\title{
CCLA: an accurate method and web server for cancer cell line authentication using gene expression profiles
}

Qiong Zhang", Mei Luo ${ }^{\#}$, Chun-Jie Liu, An-Yuan Guo*

Department of Bioinformatics and Systems Biology, Key Laboratory of Molecular Biophysics of the Ministry of Education, Hubei Bioinformatics and Molecular Imaging Key Laboratory, College of Life Science and Technology, Huazhong University of Science and Technology, Wuhan, 430074, China.

Running title: CCLA: a cancer cell line authentication method

${ }^{\#}$ Equal contribution

* Correspondence: An-Yuan Guo

Email: guoay@hust.edu.cn

Department of Bioinformatics and Systems Biology, College of Life Science and Technology, Huazhong University of Science and Technology, 1037 Luoyu Road, Wuhan 430074, China. 


\section{Abstract}

Cancer cell lines (CCLs) as important model systems play critical roles in cancer researches. The misidentification and contamination of CCLs are serious problems, leading to unreliable results and waste of resources. Current methods for CCL authentication are mainly based on the CCL-specific genetic polymorphisms, whereas no method is available for CCL authentication using gene expression profiles. Here, we developed a novel method and homonymic web server (CCLA, Cancer Cell Line Authentication, http://bioinfo.life.hust.edu.cn/web/CCLA/) to authenticate 1,291 human CCLs of 28 tissues using gene expression profiles. CCLA curated CCL-specific gene signatures and employed machine learning methods to measure overall similarities and distances between the query sample and each reference CCL. CCLA showed an excellent speed advantage and high accuracy with a top 1 accuracy of $96.58 \%$ or $92.15 \%$ (top 3 accuracy of $100 \%$ or $95.11 \%$ ) for microarray or RNA-Seq validation data (719 samples, 461 CCLs), respectively. To the best of our knowledge, CCLA is the first approach to authenticate CCLs based on gene expression. Users can freely and conveniently authenticate CCLs using gene expression profiles or NCBI GEO accession on CCLA website.

Keywords: human cancer cell lines, cell line authentication, gene expression profiles, RNA-Seq, microarray 


\section{Introduction}

2 Cancer cell lines (CCLs) as important components offering unlimited biological

3 materials play vital roles in life science studies. CCLs could serve as excellent model

4 systems for the investigation of cancer biology, the simulation of drug response, and

5 the development of clinical treatment on cancers (Holen et al. 2017). The utilization of

6 CCLs is an effective and common practice in cancer researches (Bairoch A 2018;

7 Barretina et al. 2012). However, the misidentification and contamination of CCLs are

8 long-standing and prevalent problems (Capes-Davis and Neve 2016; Horbach and

9 Halffman 2017; Development Organization Workgroup Asn-0002 2010), which could

10 introduce erroneous, misleading, and false positive findings, and further result in

11 invalid results and waste of resources. Researchers have raised extensive awareness of

12 CCL authentication, the NIH and various journals have required cell line

13 authentication for publications (Lorsch et al. 2014; Fusenig et al. 2017; Geraghty et al.

14 2014).

15 Up to date, available methods for CCL authentication were based on the DNA

16 polymorphism information, such as short tandem repeats (STRs) and single nucleotide

17 polymorphisms (SNPs) profiling (Dirks and Drexler 2005; Demichelis et al. 2008).

18 STR profiling is the most common and standard method recommended by American

19 Type Culture Collection (ATCC) for cell line authentication (Capes-Davis et al. 2010),

20 and the SNP genotyping, either in combination with STRs or alone, was considered as

21 an alternative method (Yu et al. 2015; Freedman et al. 2015). Although the STR and 
1 SNP methods had been widely used to authenticate CCLs in the past decades, they need

2 additional experiments and could not be directly applied on expression data. Even

3 though several methods (e.g., CeL-ID) utilized RNA-Seq data to authenticate CCLs

4 (Fasterius et al. 2017; Mohammad et al. 2019; Strong et al. 2014), their core algorithms

5 still retrieved CCL-specific DNA polymorphism from RNA-Seq reads, which

6 barricaded the application on gene expression data and required professional

7 bioinformatics skills (e.g., SNP calling, polymorphism matching, and threshold). Thus,

8 a convenient and precise tool using gene expression profiles for CCL authentication is

9 an urgent requirement and will benefit the scientific reproducibility.

10 CCLs with similar genomic information have various expression profiles, which

11 results in distinct characteristics for different CCLs (Domcke et al. 2013). The

12 specifically expressed genes (SEGs), which were expressed in a unique or a small

13 number of conditions, could serve as molecular features for different CCLs(Goodspeed

14 et al. 2016; Zhang et al. 2018), and provide important clues for the CCL authentication.

15 High-throughput transcriptome technologies including RNA-Seq and microarray have

16 offered numerous expression data of CCLs, such as the Genomics of Drug Sensitivity

17 in Cancer (GDSC) (Garnett et al. 2012), Cancer Cell Line Encyclopedia (CCLE)

18 (Ghandi et al. 2019), Harmonizome (Rouillard et al. 2016), and others etc. (Klijn et al.

19 2015a; Hollingshead et al. 2014). These data provided convenience for the SEGs and

20 marker genes detection in CCLs, and laid the foundation to develop methods for CCL

21 authentication using gene expression profiles. Moreover, gene expression profiles

22 based CCL authentication methods could bypass the procedure of DNA polymorphism 
1 calling, and benefit the authentication of CCLs which lack DNA information (e.g.

2 transcriptional studies, gene function analysis, microarray data, and difficult to

3 re-access the original cell lines etc.).

$4 \quad$ In this study, we developed a novel method and web server named CCLA (Cancer

5 Cell Line Authentication), which combined machine learning methods and single

6 sample gene-set enrichment analysis (ssGSEA) algorithm to authenticate 1,291 CCLs

7 using gene expression profiles from RNA-Seq or microarray platform. Our evaluation

8 results demonstrated that CCLA could rapidly and precisely authenticate CCLs.

\section{Results}

\section{The summary of CCLA method}

The workflow of CCLA is represented in the Figure 1 and the detailed algorithm is

12 illustrated in the method section. In brief, CCLA integrated gene expression profiles

13 and machine learning algorithms to authenticate the potential belonging for CCLs

14 (Figure 1): 1) ssGSEA scores of signature gene sets were used as signatures for CCLs to

15 replace the raw gene expression profiles, which could show a more robust pattern and

16 avoid the severe bias of expression profiles from different sources; 2) A prediction

17 model built by random forest (RF) algorithm was employed to pre-classify the query

18 sample into a candidate category based on ssGSEA scores of signature genes; 3 ) After

19 the categorization procedure, CCLA calculated the overall similarities and distances

20 between the query sample and each reference CCL in the candidate category. Finally, 
1 the top 5 reference CCLs with the highest correlations and the least distances were

2 considered as the potential belongings for the query sample.

\section{Accuracy and feasibility assessment for CCLA on public datasets}

To evaluate the performance of CCLA on CCL authentication, we used other

5 datasets as test data which were independent of the reference one. We applied CCLA

6 on three kinds of gene expression datasets from RNA-Seq and microarray platforms

7 (Table 1), including: 1) Public untreated CCLs from different laboratories; 2)

8 Different passages and treatments of CCLs; 3) Well-known or published incorrect and

9 misidentified CCLs. In total, 719 samples of 461 CCLs from 15 individual studies

10 were enrolled in this evaluation, including 573 samples of 456 CCLs from RNA-Seq

11 technology and 146 samples of 14 CCLs from microarray platform (Table 1 and

12 Supplementary Table S1). Among them, 511 samples were from GDSC database or

13 E-MTAB-2706 dataset, which were shared by more than one sources. For example,

14 the expression data of CCL "HCT15" were deposited in three databases, and the

15 expression data in the CCLE database would be used as the reference profile, while

16 the records in other two databases were worked as test data to assess the performance

17 of CCLA. The confidence of CCLA results was mainly evaluated by the distributions

18 of expressed signature genes in the query sample and resulting reference CCLs: 1) The

19 profiles of expressed signature genes in the query sample and reference CCLs (Figure

$202 \mathrm{~A}, 2 \mathrm{~B}) ; 2$ ) The distribution of gene signatures in the query sample and the resulting

21 reference CCLs (Figure 2C). 
1 As expected, CCLA showed a remarkable authentication power on CCLs both in

2 the RNA-Seq and microarray datasets. Generally, CCLA achieved a high accuracy of

$396.58 \%$ or $92.15 \%$ for the top 1 CCL (target CCL ranking the first one) on microarray

4 or RNA-Seq data, respectively, while considering results in the top 3 list, the accuracy

5 of CCL authentication was increased to $100 \%$ or $95.11 \%$ (Table 1 and Supplementary

6 Table S1). The validation datasets for CCLA evaluation were widely spread in

7 approximate 100 cancer types, suggesting that the power of CCLA was not limited in

8 a small number of conditions.

9 Moreover, we wondered whether the number of reference CCLs per tissue could

10 affect the authentication power of CCLA, and then investigated the relationship

11 between the accuracy of CCLA and the number of reference CCLs in tissues (Figure

12 2D, Supplementary Table S2). To avoid the bias caused by the sample size of

13 validation datasets, tissues (organs) with validation sample size more than 10 were

14 enrolled in this evaluation. Notably, CCLA showed excellent performances

15 (considering the top1, top3, top5 accuracy, respectively) on tissues containing

16 different numbers (from 11 to 143) of reference CCLs (Figure 2D). The accuracy of

17 CCLA showed a slight difference between tissues (no statistical significance) and did

18 not increased (or decreased) with the number of reference CCLs in tissues (Figure

19 2D), suggesting there is no correlation between the number of reference CCLs per

20 tissue and the accuracy of CCLA (Figure 2D, Pearson correlation coefficient $<0.27$,

$21 \quad$ P-value > 0.4). 

passages and treatments. The RNA-Seq dataset GSE111485 from GEO database

3 containing 18 HeLa samples of different conditions [controls $(n=12), 7$ passages $(n=$

$43)$ and 50 passages $(n=3)]$ from different laboratories was employed to evaluate the

5 authentication power of CCLA on CCLs with different passages. Although the

6 passage times could influence the stability of genome and transcriptional profiles for

7 CCLs(Liu et al. 2019), CCLA still showed a robust power on CCLs from different

8 passages and laboratories. All of the $18 \mathrm{HeLa}$ samples, no matter where they from and

9 how many passages, were accurately authenticated as HeLa-original lines by CCLA

10 (Supplementary Table S1). In addition, CCLA can perform well on expression data of

11 CCLs under different treatments including drug treatment, gene over-expression, and

12 microRNA transfection treatments etc. (Table 1). For example, 133 samples of CCLs

13 treated by drugs from 6 independent studies were accurately authenticated as the

14 original ones by CCLA (100\% accuracy for top 3 results, Table 1), while the accuracy

15 was slightly decreased in the samples from GDSC database (87.50\% accuracy for 122

16 CCLs with drug treatment). Besides, CCLs with gene over-expression (GSE61692

17 and GSE23655) or gene knockout (GSE101966) treatments were all correctly

18 authenticated as the original ones by CCLA (Table 1, Supplementary Table S1).

19 Furthermore, we also assessed the power of CCLA on the well-known

20 misidentified CCLs, such as the MDA-MB-435 cell line, which was not a human

21 breast cancer cell line but had been proved as M14 melanoma cell line by ATCC and

22 several laboratories (Christgen and Lehmann 2007; Lacroix 2009; Prasad and Gopalan 
1 2015). Interesting, the authentication for 8 MDA-MB-435 cell line samples

2 (GSE128624) by CCLA showed that all of them were melanoma cell lines

3 (Supplementary Table S3), implying the misidentification of MD-AMB-435 cell line

4 was a long-time event and CCLA could serve as a valuable tool to benefit the

5 reproducibility of scientific data and results based on the available expression data.

\section{Comparison with other approaches}

$7 \quad$ Although a few methods (e.g., CeL-ID and Fasterius' method) could utilize

8 RNA-Seq data to authenticate CCLs (Fasterius et al. 2017; Mohammad et al. 2019),

9 their core algorithms retrieved genomic polymorphism of samples from RNA-Seq

10 reads (not the expression profiles) to match CCL-specific SNPs and could not be

11 applied on microarray data. Meanwhile, these methods just stated a pipeline and did not

12 provide any mature software (package, tool or online server) and important parameters

13 (e.g. the version of used tools, the match pattern, the reference SNPs of CCLs, and the

14 threshold etc.) in their publications, which made it very difficult to reproduce their

15 results. Thus, we just compared the authentication results of CCLA and CeL-ID based

16 on the same RNA-seq data used by CeL-ID (Table 2).

17 Two datasets containing 20 samples (12 samples of MCF7 CCL from GSE23655,

188 samples of HCT116 CCL from GSE101966) were enrolled to benchmark the

19 performance of CCLA and CeL-ID. We first processed the RNA-Seq data to obtain

20 gene expression profiles of CCLs according to the HISAT2-StringTie protocol (Pertea

21 et al. 2016), and then applied CCLA to authenticate them. All samples in GSE101966

22 dataset were authenticated as HCT116-orignal cell lines, while samples of MCF7 cell 
1 line were authenticated as MCF7 as well. Thus, our benchmark results suggested that

2 CCLA showed a similar accuracy as the method CeL-ID using DNA polymorphism

3 from RNA-Seq data (Table 2). Moreover, CCLA showed several excellent advantages

4 on time and convenience (time: a few seconds for CCLA, much time cost for SNP

5 calling in CeL-ID; cost: free; precondition: only need gene expression profiles for

6 CCLA, several bioinformatics tools need in CeL-ID; polymorphism loss: none for

7 CCLA, always issues for polymorphism based methods; and etc.). Furthermore, our

8 CCLA is the only available tool and online web server to provide mature and

9 convenient service for CCL authentication using gene expression profiles.

\section{Website interface of CCLA}

11 For the convenient application of CCLA by users, we developed a homonymic web

12 server to provide free service of 1,291 CCLs authentication (Figure 3). Users could

13 easily authenticate and assess their interested CCLs using gene expression data. CCLA

14 accepts a NCBI GEO accession of microarray data or unfiltered gene expression matrix

15 (Figure 3A), whose rows represent the normalized expression value for genes (FPKM,

16 RPKM and TPM format for RNA-Seq data, while RMA and MAS5 for microarray data)

17 and columns are samples. Once the target CCL is selected (Figure 3A), CCLA provides

18 an overall view of outputs and evidence for the authentication of query samples (Figure

19 3B). For example, an individual page displays the detailed results: 1) The top five

20 candidate CCLs for each query sample (Figure 3C); 2) The profiles of expressed

21 signature genes in the query sample and reference CCLs (Figure 2A, 2B); 3) The gene

22 signal distribution of the query sample and the resulting reference CCL to the query 
1 sample evaluated by Pearson correlation and cosine distance (Figure 2C); 4) The

2 expression pattern of each signature gene in the query sample and the resulting

3 reference CCL (Figure 3D). Our CCLA is freely available at

4 http://bioinfo.life.hust.edu.cn/web/CCLA/.

\section{Discussion}

6 CCLs derived from human cancers are important biomaterials for cancer biology

7 exploration, pre-clinical modeling, clinical application and drug validation (Goodspeed

8 et al. 2016; Wilding and Bodmer 2014). The misidentification and mislabeling of CCLs

9 are long-standing and widespread problems in biomedical researches for decades

10 (Vaughan et al. 2017; Christgen and Lehmann 2007; Jäger et al. 2013), and large-scale

11 cross-contaminations and misidentification of CCLs were reported recently (Horbach

12 and Halffman 2017; Strong et al. 2014; Teixeira da Silva 2018; Rebouissou et al. 2017;

13 Bairoch 2018). However, available methods for CCL authentication were based on

14 DNA polymorphism, which could not be well applied on the transcriptome datasets and

15 be too cumbersome for biomedical researchers. To address these concerns, we

16 developed CCLA using gene expression profiles to rapidly authenticate CCLs with

17 high accuracy and robustness. Furthermore, we built a homonymic web server to

18 provide free $\quad$ CCL authentication for researchers

19 (http://bioinfo.life.hust.edu.cn/web/CCLA/).

20 The authentication of cell lines is a key factor for the reliability of biomedical

21 researches, which is required for the grant application and manuscript publication 
1 (Lorsch et al. 2014; Potash and Anderson 2009). DNA polymorphism (e.g. STRs and

2 SNPs) based approaches for CCL authentication analyze the similarities between the

3 query sample and reference CCLs in specific loci. Even there are high-throughput

4 sequencing data for CCLs, the loci-specific polymorphisms needed for CCL

5 authentication were often omitted from sequencing or quality control procedures or

6 uncertain RNA editing (Mohammad et al. 2019; Capes $\square$ Davis et al. 2013; Richards et

$7 \quad$ al. 2015; Otto et al. 2017), and different sequencers or different variant calling pipelines

8 may generate substantial disagreement results (Hwang et al. 2015, 2019; Coudray et al.

9 2018). Meanwhile, due to the severe genomic instability of CCLs and heterogeneous

10 NGS profiles, the coincidence of genetic polymorphism from different laboratories and

11 research projects was less than expected (Hudson et al. 2014; Alkan et al. 2011), thus

12 different algorithms or workflow designs for the authentication of the same CCL were

13 required. Moreover, the excess passages and environmental conditions (e.g. drug

14 exposure) could lead to the acceleration of genetic drift and alteration of alleles

15 information for CCLs, which may require special algorithms and interpretation for the

16 profiles of STR or SNP from unstable CCLs (Eltonsy et al. 2012; Marx 2014) and pose

17 another challenge for the authentication methods using DNA polymorphism (Eltonsy et

18 al. 2012). Additionally, large number of CCLs used in previous studies were focused on

19 the functional study of genes or pathways and the alterations of transcriptional profiles

20 under specific conditions, which lacked enough genomic polymorphisms for CCL

21 authentication (e.g. microarray and RNA-Seq data). Our CCLA implemented GSVA

22 algorithm to calculate a robust signal score matrix for CCLs, and then employed 
1 machine learning approaches to further identify the belongings of input dataset. Instead

2 of a fixed panel of limited number of STRs or SNPs, CCLA utilized a stable signal

3 matrix of gene expression profiles to represent CCLs and avoid the bias caused by

4 different passages of CCLs and the genome instability (Table 1), which in turn

5 strengthen the authentication power on the experimental treated CCLs (Table 1).

6 CCLA achieved an excellent authentication power for CCLs both on the RNA-Seq

7 and microarray data (Table 1). The results of CCLA from comprehensive validation

8 data (719 samples of 461 CCLs in 21 tissues from 15 independent datasets)

9 demonstrated that CCLA could authenticate CCLs with high precision: $92.15 \%$

10 (528/573), 95.11\% (545/573) of top1, top3 accuracy for RNA-Seq data; $96.58 \%$

11 (141/146), 100\% (146/146) of top1, top3 accuracy for microarray data (Table 1).

12 Furthermore, CCLA performed well on CCLs with different passages or drugs or gene

13 manipulation treatments (Table 1), suggesting the robustness of CCLA on expression

14 data of various treatments. Additionally, our validation results showed that CCLA had

15 a good sensitivity and accuracy on distinguishing CCLs from the same tissue origin

16 (Figure 2D and Supplementary Table S2). In this way, CCLA is an essential tool to

17 integrate metadata and ensure the reproducibility and reliability of results from cancer

18 research using CCLs of previous studies.

19 Although CCLA showed a high accuracy for the authentication of 1,291 CCLs, the

20 contamination (such as mixed with other cell lines and the Mycoplasma) remained a

21 serious problem uncovered in this study. The issue of contamination with other cell

22 lines often exists without obvious signs in experiments, and could result in global 
1 alteration of signal scores for the donor cell line. Considering the core algorithm,

2 CCLA may not perform well on the cross-contamination conditions, while the DNA

3 polymorphism based methods may be a better choice for this case. The contamination

4 of Mycoplasma could influence cell metabolism and growth, induce chromosomal

5 abnormalities, and alter transcriptome profiles (Geraghty et al. 2014; Olarerin-George

6 and Hogenesch 2015). Our results demonstrated that $\sim 30 \%(21 / 60)$ CCLs with

7 low-level Mycoplasma contamination were identified to their original ones, whereas

8 nearly $70 \%$ (39 out of 60) CCLs with severe Mycoplasma contamination were

9 authenticated as others (Supplementary Table S4). One possible reason is that the

10 expression patterns of CCLs with severe Mycoplasma contamination were significantly

11 changed, which was reported by previous studies (Olarerin-George and Hogenesch

12 2015; Zhang et al. 2006). In this manner, CCLs with severe contamination of

13 Mycoplasma may be authenticated as different one by CCLA, and CCLA could serve

14 as an indirect approach to imply the contamination of mycoplasma (or perhaps used

15 the wrong CCL). Finally, CCLA consolidated 1,291 commonly used CCLs in this

16 version and we will keep updating with the increase of standard datasets. No a single

17 method could provide all of the information for human cell line authentication

18 (Development Organization Workgroup Asn-0002 2010), and our CCLA could

19 represent the valuable candidate to identify CCLs on gene expression data.

20 The authentication of CCLs is an essential issue to avoid fake data and ensure the

21 scientific reproducibility and credibility. Although DNA polymorphism profiling based

22 methods are recommended for CCL authentication (Development Organization 
1 Workgroup Asn-0002 2010), the cost and inconvenience of these methods and the

2 physical re-access for the original CCLs appear as main roadblocks for their universal

3 applications on CCLs of previous studies (Freedman et al. 2015). Our transcriptome

4 profiles based method CCLA could be an important supplemental approach and new

5 direction. Additionally, most of available methods were not user-friendly for

6 researchers because they need extra bioinformatics and programming skills. Our

7 CCLA offered a convenient web server for the scientific community to rapidly

8 authenticate CCLs and valuable references for journals with less time, money and effort,

9 and even shed new light for the transcriptome profiles based cell line authentication.

\section{Conclusion}

11 In summary, CCLA is freely available and will largely contribute to the decrease of

12 CCLs misidentification. To the best of our knowledge, CCLA is the first approach and

13 the first online website to authenticate CCLs using gene expression data. CCLA can

14 serve as a useful resource for cancer research and improve the reliability of

15 biomedical results. 


\section{Materials and Methods}

\section{Collection for gene expression profiles of non-redundant reference \\ 3 cancer cell lines (CCLs)}

$4 \quad$ To obtain the relatively unbiased and authoritative gene expression profiles of

5 reference CCLs, we curated the RNA-Seq gene expression profiles of CCLs from 3

6 generally recognized CCL resources: 1) Cancer Cell Line Encyclopedia (CCLE),

7 which contains expression profiles of 934 CCLs from RNA-Seq data(Ghandi et al.

8 2019); 2) Genomics of Drug Sensitivity in Cancer (GDSC), which deposits the

9 expression profiles of 457 CCLs from RNA-Seq data(Yang et al. 2013); 3) The

10 E-MTAB-2706 dataset, which is a comprehensive transcriptional portrait of 675

11 common human CCLs (Klijn et al. 2015b).

12 Furthermore, we examined the integrity of information for all the CCLs above.

13 Briefly, all the introductions of reference CCLs were retrieved using an in-house "web

14 crawler" script programmed by the python language and its libs (e.g. urllib,

15 BeautifulSoup, and requests etc.). First, CCLs with a similar character string (e.g.

16 "HCT 116" or "HCT116" or "HCT-116" or "HCT_116", but not limited in this style)

17 and the same origin (e.g. from the colon or large-intestine etc.) were deemed as the

18 same kind of a CCL with different aliases. Then CCLs with similar origins but

19 large-distance of their names (20\%, e.g., the character difference between SW1417

20 and SW1463, not limited in this situation) were carefully checked and manually

21 examined from the webpages of the resources. In addition, when a CCL was stored in 
1 more than one source, the priority of its gene expression profile as a reference in

2 CCLA was ranked by the following order (CCLE > GDSC > E-MTAB-2706). For

3 example, the CCLE and GDSC databases simultaneously collected the expression

4 profiles of HCT116 CCL, and in this case, the gene expression profile of HCT116

5 CCL in the CCLE resource was served as a reference CCL, while the one in the

6 GDSC was used as a validation sample for HCT116 CCL. Based on the above

7 procedures, 1,471 kinds of non-redundant or unique reference CCLs (883 CCLs from

8 CCLE database, 391 from GDSC, and 146 from E-MTAB-2706) were kept for further

9 analyses.

10 Curation of signature genes for CCLs

11 First, the gene signatures of each CCL were retrieved from literature mining,

12 resource collection and de novo detection processes: 1) Literature mining from

13 publications. In this process, we used several key words (e.g., "maker gene",

14 "specifically expressed gene (SEG)", and "highly expressed gene" etc.) in the

15 PubMed database to retrieve candidate signature genes for corresponding CCLs; 2)

16 Resource collection. Two databases Harmonizome and SEGreg (Rouillard et al. 2016;

17 Tang et al. 2018) were the main resources to collect the signature genes. In

18 Harmonizome, those candidate signature genes with a score $>1$ were used, which

19 indicates that the gene has a strong positive gene-CCL association. In SEGreg

20 database, genes with the tag "high" in the corresponding CCL was deemed as

21 candidate signature genes; 3) De novo detection, SEGs were detected using SEGtool

22 (Zhang et al. 2018) (default parameters, p-value $<=0.05$, highly expressed pattern) on 
1 gene expression profiles of 1,471 reference CCLs, and the output SEGs were acted as

2 candidate signature genes as well.

3 Second, candidate signature genes from the above three processes were integrated

4 to explore putative signature genes by the following two steps: 1) For CCLs from the

5 same tissue (or organ), we calculated and adjusted the ratio of tissue-specific genes to

6 candidate signature genes. For example, if the ratios of tissue-specific genes (with the

7 number of 30) were more than $40 \%$ in 5 CCLs, we randomly assigned the same

8 number of tissue-specific genes (e.g., the number is $30 / 5=6$ in this case, allowed $1 / 5$

$9=20 \%$ repetition) to the 5 CCLs; 2) For CCLs with similar candidate signature genes,

10 we implemented the same operation as the step 1. Furthermore, we measured the

11 reliability of signature genes in reference CCLs by examining their expression levels.

12 After the above processes, the retained genes were considered as putative signature

13 genes. Finally, 180 out of 1,471 CCLs that did not have enough signature genes (less

14 than 50) were dropped, and the rest 1,291 reference CCLs were kept for further 15 analyses.

16 Signature calculation and model construction for CCLs

17 To avoid the bias and technical variability of gene expression caused by the noise,

18 different quantile normalization methods, and various experiment treatments, ssGSEA

19 algorithm was implemented to calculate the enrichment scores of signature genes for

20 each CCL, which could serve as robust expression features compared with the raw gene

21 expression profiles (Figure 1). Thus, the raw expression profiles of reference CCLs

22 have been represented by ssGSEA scores of signature genes sets, and each CCL has the 
1 same 1,291 signatures, whose expression values are ssGSEA scores. Next, we

2 constructed a 1,291 x 1,291 signature matrix for the reference CCLs, in which, each

3 row is the corresponding signature values in 1,291 CCLs, and columns represent CCLs.

4 Furthermore, t-distributed stochastic neighbor embedding (t-SNE) algorithm was

5 used for the classification and clustering of reference CCLs based on their signatures

6 (parameters: dims $=3$, perplexity $=50$, max_iter $=5000$, theta $=0$, pca $=$ TRUE), and

7 three groups were obtained. Subsequently, we employed the random forest (RF)

8 algorithm to extract features from reference CCLs with their group labels determined

9 by t-SNE (the importance of each feature was represented in the Supplementary Table

10 S5), and then built a prediction model that would been applied to estimate the potential

11 group for the query CCL (Figure 1).

\section{CCL authentication}

13 In order to accurately authenticate CCLs, CCLA calculates the ssGSEA score of

14 signature genes for the query CCL, then applies the prediction model (built by RF

15 algorithm in the model construction step above) to pre-classify the candidate group of

16 the query CCL (Figure 1). Then CCLA employs Pearson correlation and cosine

17 distance to measure the similarities and divergences between the query CCL and each

18 reference CCL in the pre-classified category. Then, CCLA ranked reference CCLs in

19 the given category by Pearson correlation coefficient and cosine distance. The

20 reference CCL with the highest similarity and least divergence was considered as the

21 putative belonging of the query CCL, and the top 5 CCLs were also listed as candidate

22 results. 


\section{Validation data collection}

2 Both gene expression profiles of CCLs from RNA-Seq and microarray platforms

3 were curated to evaluate the accuracy of CCLA. Three kinds of CCLs with gene

4 expression profiles were collected: 1) Public untreated CCLs from different

5 laboratories; 2) Different passages and treatments of CCLs; 3) Well-known

6 misidentified CCLs (Table 1).

7 We employed the following criteria to judge a successful authentication in CCLA:

8 1) The consistency between paper declared CCL and the results of CCLA. For example,

9 if a CCL was identified as another one by CCLA which was different from the original

10 paper, we deemed this as an inaccuracy authentication, otherwise is correct, expect for

11 the well-known misidentified or contaminated CCLs (such as MDA-MB-435 cell line,

12 the American Type Culture Collection (ATCC) reported that the MDA-MB-435 cell

13 line is not breast cancer but actually melanoma related cell line); 2) For the well-known

14 misidentified CCLs (e.g. MDA-MB-435 cell line), all the MDA-MB-435 strains were

15 considered as the melanoma cell lines, and if any MDA-MB-435 cell line was

16 identified as the melanoma origin, we deemed this authentication was a correct case. 


\section{Abbreviations}

CCLA: cancer cell line authentication

CCL: cancer cell line

GDSC: Genomics of Drug Sensitivity in Cancer

CCLE: Cancer Cell Line Encyclopedia

CHCC: common human cancer cell

EBI: European Bioinformatics Institute

FPKM: fragments per kilobase per million mapped fragments

RPKM: reads per kilobase per million mapped reads

TPM: transcripts per kilobase per million mapped reads

ssGSEA: single sample gene-set enrichment analysis

SEG: specifically expressed gene

SNP: single nucleotide polymorphism

STR: short tandem repeat

\section{Competing interests}

The authors declare that they have no competing interests.

\section{Authors' contributions}

Q.Z and M.L: Methodology, Data collection, Webserver work, and Manuscript writing; M.L, Q.Z and C.J.L: bioinformatics analysis; AG and QZ: Conceptualization, Writing, Revising, Funding Acquisition, and Supervision. 


\section{Acknowledgements}

We would like to thank colleagues for data production and web server testing. We are also grateful to our users and all members of our lab for their valuable suggestions and comments.

We also acknowledge the research funding from the National Key Research and Development Program of China (Grant No. 2017YFA0700403), National Natural Science Foundation of China (NSFC) (Grant Nos. 31822030, 31801113, and 31771458), China Postdoctoral Science Foundation (Grant No. 2018M632830 and 2019M652623), the Fundamental Research Funds for the Central Universities (2018KFYRCPY002) and the program for HUST Academic Frontier Youth Team. 


\section{Reference}

Alkan C, Coe BP, Eichler EE. 2011. Genome structural variation discovery and genotyping. Nat Rev Genet 12: 363-376.

Bairoch A. 2018. The Cellosaurus, a Cell-Line Knowledge Resource. J Biomol Tech JBT 29: 25-38.

Barretina J, Caponigro G, Stransky N, Venkatesan K, Margolin AA, Kim S, Wilson CJ, Lehár J, Kryukov GV, Sonkin D, et al. 2012. The Cancer Cell Line Encyclopedia enables predictive modeling of anticancer drug sensitivity. Nature 483: 603.

Capes-Davis A, Neve RM. 2016. Authentication: A Standard Problem or a Problem of Standards? PLOS Biol 14: e1002477.

Capes $\square$ Davis A, Reid YA, Kline MC, Storts DR, Strauss E, Dirks WG, Drexler HG, MacLeod RAF, Sykes G, Kohara A, et al. 2013. Match criteria for human cell line authentication: Where do we draw the line? Int J Cancer 132: 2510-2519.

Capes-Davis A, Theodosopoulos G, Atkin I, Drexler HG, Kohara A, MacLeod RAF, Masters JR, Nakamura Y, Reid YA, Reddel RR, et al. 2010. Check your cultures! A list of cross-contaminated or misidentified cell lines. Int J Cancer 127: $1-8$.

Christgen M, Lehmann U. 2007. MDA-MB-435: the questionable use of a melanoma cell line as a model for human breast cancer is ongoing. Cancer Biol Ther 6: $1355-1357$.

Coudray A, Battenhouse AM, Bucher P, Iyer VR. 2018. Detection and benchmarking of somatic mutations in cancer genomes using RNA-seq data. PeerJ 6: e5362.

Demichelis F, Greulich H, Macoska JA, Beroukhim R, Sellers WR, Garraway L, Rubin MA. 2008. SNP panel identification assay (SPIA): a genetic-based assay for the identification of cell lines. Nucleic Acids Res 36: 2446-2456.

Development Organization Workgroup Asn-0002 ATCCS. 2010. Cell line 
misidentification: the beginning of the end. Nat Rev Cancer 10: 441-448.

Dirks WG, Drexler HG. 2005. Authentication of scientific human cell lines: easy-to-use DNA fingerprinting. Methods Mol Biol Clifton NJ 290: 35-50.

Domcke S, Sinha R, Levine DA, Sander C, Schultz N. 2013. Evaluating cell lines as tumour models by comparison of genomic profiles. Nat Commun 4: 2126.

Eltonsy N, Gabisi V, Li X, Russe KB, Mills GB, Stemke-Hale K. 2012. Detection algorithm for the validation of human cell lines. Int J Cancer 131: E1024-1030.

Fasterius E, Raso C, Kennedy S, Rauch N, Lundin P, Kolch W, Uhlén M, Al-Khalili Szigyarto C. 2017. A novel RNA sequencing data analysis method for cell line authentication ed. M. Costa. PLOS ONE 12: e0171435.

Freedman LP, Gibson MC, Ethier SP, Soule HR, Neve RM, Reid YA. 2015. Reproducibility: changing the policies and culture of cell line authentication. Nat Methods 12: 493-497.

Fusenig NE, Capes-Davis A, Bianchini F, Sundell S, Lichter P. 2017. The need for a worldwide consensus for cell line authentication: Experience implementing a mandatory requirement at the International Journal of Cancer. PLOS Biol 15: e2001438.

Garnett MJ, Edelman EJ, Heidorn SJ, Greenman CD, Dastur A, Lau KW, Greninger P, Thompson IR, Luo X, Soares J, et al. 2012. Systematic identification of genomic markers of drug sensitivity in cancer cells. Nature 483: 570-575.

Geraghty RJ, Capes-Davis A, Davis JM, Downward J, Freshney RI, Knezevic I, Lovell-Badge R, Masters JRW, Meredith J, Stacey GN, et al. 2014. Guidelines for the use of cell lines in biomedical research. Br J Cancer 111: 1021-1046.

Ghandi M, Huang FW, Jané-Valbuena J, Kryukov GV, Lo CC, McDonald ER, Barretina J, Gelfand ET, Bielski CM, Li H, et al. 2019. Next-generation characterization of the Cancer Cell Line Encyclopedia. Nature 569: 503-508. 
Goodspeed A, Heiser LM, Gray JW, Costello JC. 2016. Tumor-Derived Cell Lines as Molecular Models of Cancer Pharmacogenomics. Mol Cancer Res MCR 14: $3-13$.

Holen I, Speirs V, Morrissey B, Blyth K. 2017. In vivo models in breast cancer research: progress, challenges and future directions. Dis Model Mech 10: $359-371$.

Hollingshead MG, Stockwin LH, Alcoser SY, Newton DL, Orsburn BC, Bonomi CA, Borgel SD, Divelbiss R, Dougherty KM, Hager EJ, et al. 2014. Gene expression profiling of 49 human tumor xenografts from in vitro culture through multiple in vivo passages--strategies for data mining in support of therapeutic studies. BMC Genomics 15: 393.

Horbach SPJM, Halffman W. 2017. The ghosts of HeLa: How cell line misidentification contaminates the scientific literature. PLOS ONE 12: e0186281.

Hudson AM, Yates T, Li Y, Trotter EW, Fawdar S, Chapman P, Lorigan P, Biankin A, Miller CJ, Brognard J. 2014. Discrepancies in Cancer Genomic Sequencing Highlight Opportunities for Driver Mutation Discovery. Cancer Res 74: 6390-6396.

Hwang K-B, Lee I-H, Li H, Won D-G, Hernandez-Ferrer C, Negron JA, Kong SW. 2019. Comparative analysis of whole-genome sequencing pipelines to minimize false negative findings. Sci Rep 9: 3219.

Hwang S, Kim E, Lee I, Marcotte EM. 2015. Systematic comparison of variant calling pipelines using gold standard personal exome variants. Sci Rep 5: 17875 .

Jäger W, Horiguchi Y, Shah J, Hayashi T, Awrey S, Gust KM, Hadaschik BA, Matsui Y, Anderson S, Bell RH, et al. 2013. Hiding in plain view: genetic profiling reveals decades old cross contamination of bladder cancer cell line KU7 with HeLa. J Urol 190: 1404-1409. 
Klijn C, Durinck S, Stawiski EW, Haverty PM, Jiang Z, Liu H, Degenhardt J, Mayba O, Gnad F, Liu J, et al. 2015a. A comprehensive transcriptional portrait of human cancer cell lines. Nat Biotechnol 33: 306-312.

Klijn C, Durinck S, Stawiski EW, Haverty PM, Jiang Z, Liu H, Degenhardt J, Mayba O, Gnad F, Liu J, et al. 2015b. A comprehensive transcriptional portrait of human cancer cell lines. Nat Biotechnol 33: 306-312.

Lacroix M. 2009. MDA-MB-435 cells are from melanoma, not from breast cancer. Cancer Chemother Pharmacol 63: 567-567.

Liu Y, Mi Y, Mueller T, Kreibich S, Williams EG, Van Drogen A, Borel C, Frank M, Germain P-L, Bludau I, et al. 2019. Multi-omic measurements of heterogeneity in HeLa cells across laboratories. Nat Biotechnol 37: 314-322.

Lorsch JR, Collins FS, Lippincott-Schwartz J. 2014. Fixing problems with cell lines. Science 346: 1452-1453.

Marx V. 2014. Cell-line authentication demystified. Nat Methods 11: 483-488.

Mohammad TA, Tsai YS, Ameer S, Chen H-IH, Chiu Y-C, Chen Y. 2019. CeL-ID: cell line identification using RNA-seq data. BMC Genomics 20: 81.

Olarerin-George AO, Hogenesch JB. 2015. Assessing the prevalence of mycoplasma contamination in cell culture via a survey of NCBI's RNA-seq archive. Nucleic Acids Res 43: 2535-2542.

Otto R, Sers C, Leser U. 2017. Robust in-silico identification of cancer cell lines based on next generation sequencing. Oncotarget 8: 34310-34320.

Pertea M, Kim D, Pertea GM, Leek JT, Salzberg SL. 2016. Transcript-level expression analysis of RNA-seq experiments with HISAT, StringTie and Ballgown. Nat Protoc 11: 1650-1667.

Potash J, Anderson KC. 2009. What's Your Line? Clin Cancer Res 15: 4251-4251.

Prasad VV, Gopalan RO. 2015. Continued use of MDA-MB-435, a melanoma cell 
line, as a model for human breast cancer, even in year, 2014. Npj Breast Cancer 1: 15002.

Rebouissou S, Zucman-Rossi J, Moreau R, Qiu Z, Hui L. 2017. Note of caution: Contaminations of hepatocellular cell lines. J Hepatol 67: 896-897.

Richards S, Aziz N, Bale S, Bick D, Das S, Gastier-Foster J, Grody WW, Hegde M, Lyon E, Spector E, et al. 2015. Standards and Guidelines for the Interpretation of Sequence Variants: A Joint Consensus Recommendation of the American College of Medical Genetics and Genomics and the Association for Molecular Pathology. Genet Med Off J Am Coll Med Genet 17: 405-424.

Rouillard AD, Gundersen GW, Fernandez NF, Wang Z, Monteiro CD, McDermott MG, Ma'ayan A. 2016. The harmonizome: a collection of processed datasets gathered to serve and mine knowledge about genes and proteins. Database 2016: baw 100 .

Strong MJ, Baddoo M, Nanbo A, Xu M, Puetter A, Lin Z. 2014. Comprehensive high-throughput RNA sequencing analysis reveals contamination of multiple nasopharyngeal carcinoma cell lines with HeLa cell genomes. J Virol 88: $10696-10704$.

Tang Q, Zhang Q, Lv Y, Miao Y-R, Guo A-Y. 2018. SEGreg: a database for human specifically expressed genes and their regulations in cancer and normal tissue. Brief Bioinform.

Teixeira da Silva JA. 2018. Incorrect cell line validation and verification. Ann Transl Med 6: 136-136.

Vaughan L, Glänzel W, Korch C, Capes-Davis A. 2017. Widespread Use of Misidentified Cell Line KB (HeLa): Incorrect Attribution and Its Impact Revealed through Mining the Scientific Literature. Cancer Res 77: 2784-2788.

Wilding JL, Bodmer WF. 2014. Cancer cell lines for drug discovery and development. Cancer Res 74: 2377-2384. 
Yang W, Soares J, Greninger P, Edelman EJ, Lightfoot H, Forbes S, Bindal N, Beare D, Smith JA, Thompson IR, et al. 2013. Genomics of Drug Sensitivity in Cancer (GDSC): a resource for therapeutic biomarker discovery in cancer cells. Nucleic Acids Res 41: D955-D961.

Yu M, Selvaraj SK, Liang-Chu MMY, Aghajani S, Busse M, Yuan J, Lee G, Peale F, Klijn C, Bourgon R, et al. 2015. A resource for cell line authentication, annotation and quality control. Nature 520: 307-311.

Zhang Q, Liu W, Liu C, Lin S-Y, Guo A-Y. 2018. SEGtool: a specifically expressed gene detection tool and applications in human tissue and single-cell sequencing data. Brief Bioinform 19: 1325-1336.

Zhang S, Tsai S, Lo S-C. 2006. Alteration of gene expression profiles during mycoplasma-induced malignant cell transformation. BMC Cancer 6: 116. 


\section{Figure legend}

Figure 1 The data resources and algorithm of CCLA. (A) The resource collection and model construction for reference CCLs. The reference data of CCLs in CCLA were from three resources: CCLE, GDSC and CHCC (E-MTAB-2706 dataset in EBI). The gene signatures of CCLs were from three parts: 1) Text mining from publications; 2) SEGs collection from databases; 3) De novo detection by R package

SEGtool. (B) The core steps of CCLA algorithm.

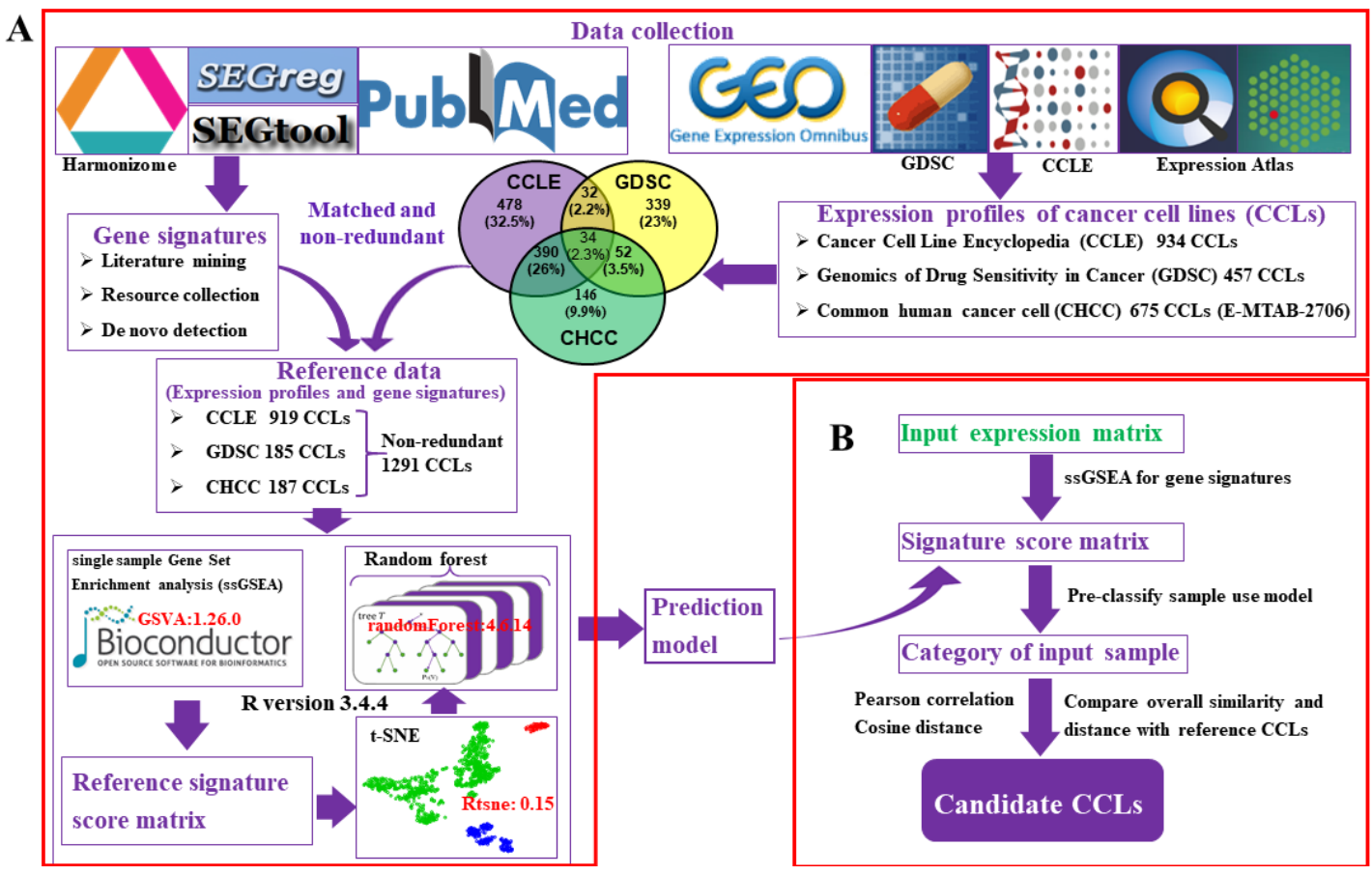


Figure 2 The accuracy assessment of CCLA. (A) The number of expressed signature genes in query sample and matched reference CCL; (B) The amount of missing signature genes in a query sample compared with the reference CCL; (C) The ssGSEA scores of signature gene sets in query sample and reference CCL. The X-axis indicates signatures of the reference CCL, while the Y-axis shows the ssGSEA scores of signature gene sets in the query sample (grey color) and candidate CCL (red color); (D) The accuracy of CCLA in different tissues. The Y-axis is the accuracy of validation datasets in corresponding tissue, and the $\mathrm{X}$-axis shows the number of validated CCLs in the tissues. The top 1 accuracy means the target CCL ranks first of the outcomes of CCLA, while the top 3(5) accuracy indicates the target CCL appears in the first 3(5) results. The "cor" means the Pearson correlation coefficient between the accuracy and the reference CCLs in tissues, where the $\mathrm{p}$ is the P-value of the correlation. The low correlation here implies the accuracy of CCLA has no dependency with the number of reference CCLs per tissue.
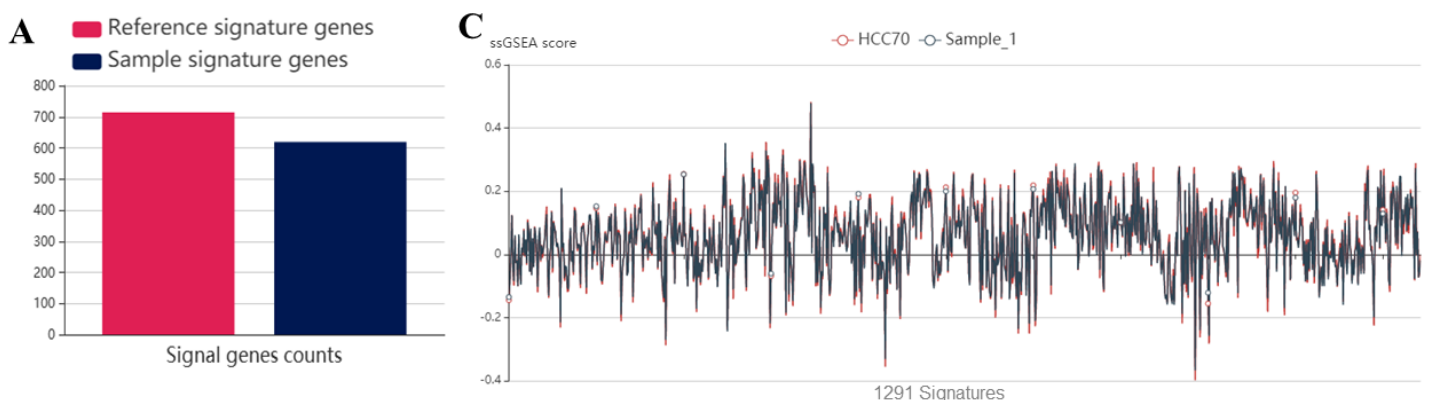

\section{B}

Missing signature genes Sample signature genes

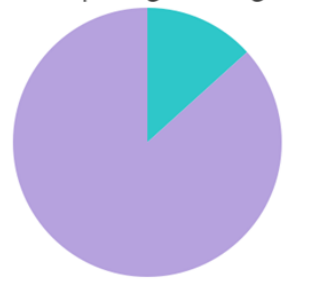

D

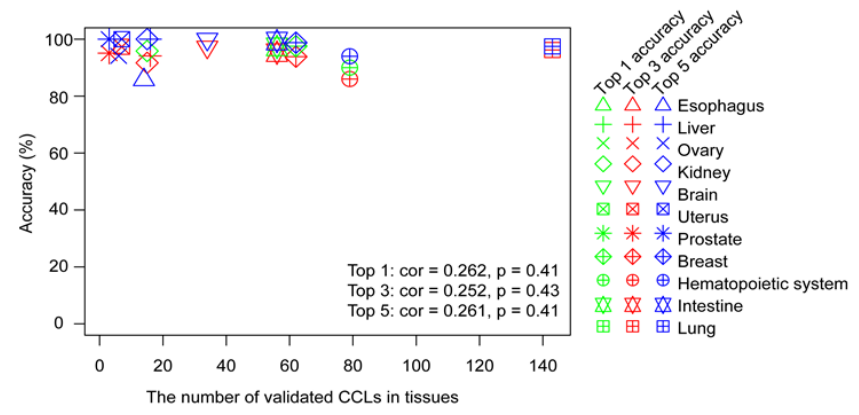


Figure 3 The interface of CCLA web server. (A) A snapshot of the authentication page; (B) A partial of the authentication result page for the query sample in CCLA web server; (C) The detail results of top 5 candidate CCLs for the query sample; (D) The expression profiles of signature genes in the query sample and the reference CCL.
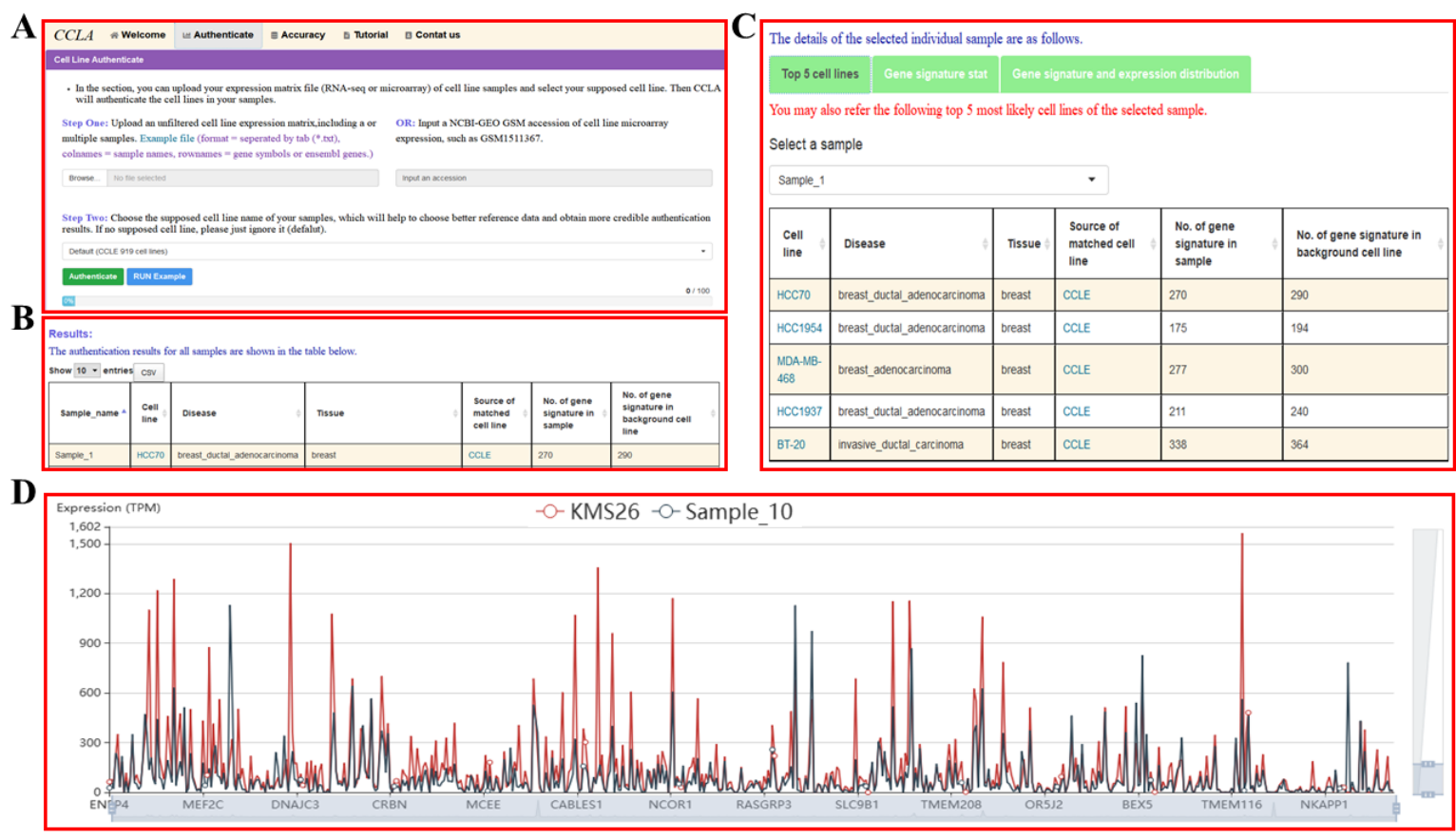
Tables

Table 1 Validation datasets and corresponding accuracies of CCLA

\begin{tabular}{|c|c|c|c|c|c|c|c|}
\hline \multirow[t]{2}{*}{ Datasets } & \multirow[t]{2}{*}{ \#samples } & \multirow[t]{2}{*}{ \#CCLs } & \multicolumn{3}{|c|}{ Accuracy (\%) } & \multirow{2}{*}{ Exp. } & \multirow{2}{*}{ Treatment } \\
\hline & & & Top1 & Top3 & Top5 & & \\
\hline E-MTAB-2706 & 399 & 399 & 93.98 & 96.49 & 97.24 & TPM & Untreated \\
\hline GDSC & 112 & 112 & 81.25 & 87.50 & 93.75 & TPM & Drug \\
\hline GSE32323 & 10 & 5 & 90.00 & 100 & 100 & Array & Drug and control \\
\hline GSE54979 & 9 & 1 & 100 & 100 & 100 & Array & Drug and control \\
\hline GSE55624 & 18 & 1 & 100 & 100 & 100 & Array & Drug and control \\
\hline GSE66837 & 12 & 1 & 100 & 100 & 100 & Array & Drug and control \\
\hline GSE73318 & 36 & 6 & 100 & 100 & 100 & RPKN & Drug and control \\
\hline GSE83654 & 48 & 3 & 93.75 & 100 & 100 & Array & Drug and control \\
\hline GSE101966 & 8 & 1 & 100 & 100 & 100 & FPKM & Knock out and contro \\
\hline GSE111485 & 18 & 1 & 100 & 100 & 100 & RPKN & Passages and control \\
\hline
\end{tabular}




\begin{tabular}{lllllll}
\hline GSE57820 & 12 & 1 & 100 & 100 & 100 & Array miR-135b overexpression \\
GSE61692 & 4 & 1 & 100 & 100 & 100 & Array Overexpression and control \\
GSE23655 & 12 & 1 & 100 & 100 & 100 & Array Overexpression and control \\
GSE65168 & 8 & 1 & 100 & 100 & 100 & Array Hypoxia and control \\
GSE7458 & 13 & 1 & 92.31 & 100 & 100 & Array Degree and control \\
\hline
\end{tabular}

\#samples: The number of samples in the dataset; \#CCLs: The number of CCLs in the study; TopX: The ratio of correct CCLs in the top X records in outcome of CCLA; Exp.: The normalization method of gene expression data used in the study; Treatment: The treatment(s) used in the study.

Table 2 The comparison of CCLA with CeL-ID

\begin{tabular}{lll}
\hline & CCLA & CeL-ID \\
\hline Data type & Expression data & SNPs from RNA-Seq reads \\
Support platform & RNA-Seq \& microarray & RNA-Seq \\
Expression data & $\mathrm{Y}$ & $\mathrm{N}$ \\
Time & $10 \mathrm{~s}$ & Excessive time \\
Pre-condition & None & Professional skills and several software \\
& & for SNP calling and pattern match \\
\hline
\end{tabular}




\begin{tabular}{lll}
\hline Convenience & Web server & No mature tool \\
Capacity & 1,291 & unknown \\
Cost & Free & Free \\
\hline
\end{tabular}




\section{Supporting Information}

Supplementary Table S1: Detailed results of authenticated CCLs for the test datasets.

Supplementary Table S2: The accuracy of CCLA on different tissues.

Supplementary Table S3: The authentication results of MD-AMB-435 datasets.

Supplementary Table S4: The authentication results of CCLs contaminated by Mycoplasma.

Supplementary Table S5: The importance of features in the predicted model from RF algorithm. 